Original Article

\title{
The effects of non-elastic taping on muscle tone in stroke patients: a pilot study
}

\author{
Ji Young Kim, PT, MS ${ }^{1)}$, Jun Sub Chung, PT, MS ${ }^{1)}$, Gwon Uk JAng, PT, PhD ${ }^{1)}$, \\ SeOl PARK, PT, $\mathrm{PhD}^{1)}$, JI Won PARK, PT, $\mathrm{PhD}^{1)^{*}}$ \\ 1) Department of Physical Therapy, College of Medical Health, Catholic University of Daegu: 13-13 \\ Hayang-ro, Hayang-eup, Gyeongsan-si, Gyeongbuk 712-902, Republic of Korea
}

\begin{abstract}
Purpose] Hemiplegia occurs when posturing with a dominant flexor tone is present in the upper limbs, thus preventing increased abnormal tone. We attempted to improve the side effects of this condition using elbow re-positioning with non-elastic tape; this method is used to modulate abnormal muscle tone in chronic hemiplegic stroke patients. [Subjects and Methods] Fourteen post-stroke patients were included in this study. Non-elastic tape was applied to the elbow joint in a spiral manner. Before and after the tape was applied, the degree of spasticity (hypertonia) was measured in the elbow flexor muscles using the Modified Ashworth Scale (MAS). Global synkinesis (GS) intensity using electromyography (EMG) was measured in the biceps brachii and triceps brachii during voluntary isometric elbow contractions of the contralateral upper limbs. [Results] Application of non-elastic tape at the elbow joint significantly changed the GS intensity, but no significant changes were found when compared with the MAS. [Conclusion] This study demonstrates that non-elastic tape can be used to decrease abnormal elbow flexor tone. The findings may be used to influence the choice of intervention regarding muscle tone and spastic elbow flexion.

Key words: Stroke, Muscle tone, Non-elastic taping
\end{abstract}

(This article was submitted Jul. 29, 2015, and was accepted Sep. 25, 2015)

\section{INTRODUCTION}

A stroke involves the rapid loss of brain function due to a disturbance in the blood vessels supplied to the brain ${ }^{1)}$. Strokes often profoundly affect upper limb stability and movement capacity. Common post-stroke motor disorders are spasticity (hypertonia) and muscle synergy that induce distinctive and complex movement patterns ${ }^{1,2)}$. Muscle synergy is the mass contraction of multiple muscle groups and occurs in the arm due to various upper extremity movements. Spasticity (hypertonia) includes disabling and persistent symptoms that affect many stroke survivors and is a motor disorder characterized by a velocity-dependent resistance in muscle tone ${ }^{3-6)}$. Muscle tone is defined as the state of activity or tension of a muscle beyond its physical properties ${ }^{7)}$. Skeletal muscle tone reflects intrinsic viscoelastic muscle properties ("passive tone," "non-reflex," or "EMG silent") and includes neurogenic factors that are activated by stimuli; it is represented mainly as the stretch reflex and is also identified as "active tone," "reflex tone," or "neurogenic tone"8). Increased muscle tone in the post-stroke hemiplegic limb often negatively interferes with functional motor recovery

Corresponding author. Ji Won Park (E-mail: mylovept@ hanmail.net)

(C2015 The Society of Physical Therapy Science. Published by IPEC Inc. This is an open-access article distributed under the terms of the Creative Commons Attribution Non-Commercial No Derivatives (by-ncnd) License $<$ http://creativecommons.org/licenses/by-nc-nd/3.0/>. of the upper limb and with correct postural control ${ }^{3,6)}$. In chronic stroke patients, flexor muscle tone often appears as a complex pattern with hypertonia, thus affecting upperextremity joints ${ }^{2}$. Therefore, appropriate management of flexor muscle tone has been an important topic in rehabilitation for stroke patients.

Several management options help to decrease muscle tone and spasticity in stroke patients, such as neuromuscular electrical stimulation ${ }^{9)}$, stretching devices ${ }^{3)}$, vibratory simulation $^{10)}$, serial cast application ${ }^{11)}$, and taping ${ }^{8,12-16)}$. In a clinical setting, several types of tape are applied directly to the skin in a special manner in order to achieve various therapeutic effects, such as improved circulation, subcutaneous lymphatic drainage, muscle facilitation or inhibition, fascia correction, and mechanical correction $\left.{ }^{4}, 17\right)$. McConnell taping was introduced in 1984 by Jenny McConnell ${ }^{18)}$, this method normally involves preparation of the skin and application of a protective undertape followed by a nonelastic, rigid overtape that applies tension to the underlying soft tissues ${ }^{17)}$. These can be worn for up to eighteen hours ${ }^{18)}$. Non-elastic taping enhances the ability to generate force ${ }^{14)}$, improves joint realignment, and leads to decreased joint reaction forces ${ }^{17}$. Non-elastic taping also immediately corrects faulty joint alignment or restricts joint range of motion $(\mathrm{ROM})^{19,20)}$. Recent studies have reported significant effects on proprioception and neuromuscular control ${ }^{18,21)}$, and nonelastic taping appears to be the rehabilitation technique of choice in the clinic.

Global synkinesis (GS), known as mirror movement, motor overflow, and contralateral irradiation, is common 
in hemiparetic subjects. When the homologous part of the opposite limb is active in hemiparetic subjects, involuntary pathological muscle activity and movements elicited at several or all of the joints can be observed ${ }^{22)}$. Brain images reveal that GS is identified by bilateral excitation of the motor cortex in which one hemisphere reduces adequate inhibitory influences on the opposite hemisphere ${ }^{23)}$. The level of GS intensity in the paretic arm is related to the functional outcome of patients with post-stroke hemiparesis and is especially dependent on contralateral elbow flexor muscle contractions $^{22}$. Even though GS is well recognized by clinicians, there is still no consensus as to whether attempts should be made to reduce the synkinetic movement.

In the present study, we attempt to replicate the effect of elbow re-positioning tape by using non-elastic tape to modulate abnormal muscle tone in chronic hemiplegic stroke patients. Muscle tone can be quantified separately by employing specific measurement techniques. We aimed to measure GS intensity using electromyography (EMG) in the paretic upper limb during elbow flexion and extension movements of the contralateral arm. We used viscoelastic components (passive tone) and the Modified Ashworth Scale (MAS) for neurogenic components (active tone) ${ }^{7,}, 8,22,23$ ). The aim of the present pilot study was to determine the influence of non-elastic taping on abnormal elbow flexor tone in patients with strokes.

\section{SUBJECTS AND METHODS}

Fourteen people with post-stroke were recruited from a pool of appropriate patients at the Rehabilitation Hospital in Changwon city. The time ranged from seventeen months to fifty-eight months; mean ages and standard deviations were $49.52 \pm 9.2$. None of the subjects with a stroke were taking any antispastic medication or had any limitations in passive range of motion. The criterion for inclusion was a diagnosis of stroke with spasticity (hypertonia) in the elbow flexors, according to the Modified Ashworth Scale ${ }^{24,25)}$; this scale quantitatively evaluates the degree of passive movement during muscle stretching and determines the degree of spasticity in stroke subjects. The scale ranges from 0 to 4: Grade 0 , Grade 1, Grade 1+, Grade 2, Grade 3 and Grade 46). Those subjects who demonstrated deficits in language, attention, or cognition were excluded from the study because of their possible inability to follow experimental instructions ${ }^{22}$. All subjects understood the purpose of this study and submitted written consent prior to their participation, in accordance with the ethical standards of the Declaration of Helsinki. They were not aware of the focus of our research interests.

Subjects were seated when the experiments were carried out. During these experiments, the subjects completed the target tasks using the joints in the unaffected extremity. The positions in performing the isometric muscle contractions were constant with the elbow flexed at $90^{\circ}$, the shoulder in slight abduction $\left(15^{\circ}\right)$, and the forearm in a neutral position $^{23)}$. The relaxed (affected) upper extremity was suspended without support parallel to the trunk ${ }^{22)}$. For these positions, each subject was seated in a comfortable chair: the back was curved, the hips and knees were positioned at approximately $90^{\circ}$ of flexion, and the ankles were flexed at $90^{\circ}$ and reached on the floor. Before and immediately after application of the non-elastic tape, we measured the degree of spasticity in the elbow flexor muscles using the Modified Ashworth Scale and measured EMG activity in the affected arm. Global synkinesis was defined operationally as the EMG activity of two elbow muscles on the affected arm during maximal isometric contralateral elbow contractions. The Delsys Triagno Wireless EMG system (Delsys Inc., Boston, MA, USA) was used to record GS activity of the bicep brachii and tricep brachii muscles; active surface electrodes were placed on the respective muscle belly ${ }^{23)}$.

The elbow hypertonic position associated with post-stroke is typically present during elbow flexion, though forearm pronation appears to be more common ${ }^{25}$. Forearm pronation is formed by the biceps, the brachioradialis, the brachialis muscles, and the pronator teres ${ }^{6}$. Application of non-elastic tape has been reported to be a useful intervention for restricting joint range of motion (ROM) or unwanted postures. We modified the placement of non-elastic tape as suggested by Martin $^{13)}$ and ElKhatib ${ }^{26}$. To inhibit hypertonic positioning and to provide joint stability with neutral alignment, nonelastic tape applied in a serpentine fashion along the elbow when flexed slightly and to the forearm when in supination (Fig. 1).

A strip of hypoallergenic tape (Endura Fix Tape, EnduraTape Pty. Ltd., Australia) was applied under the non-elastic tape to minimize the effects on the skin. The hypoallergenic tape was placed from the anterior surface of the upper third of the ulna and moved in an upward spiral past the posterior aspect of the elbow joint. The tape continued in the same manner, passing and ending at the distal lateral border of the humerus. A manual was placed below the elbow joint with the intention of maintaining the elbow joint extension and forearm supination positions while a strip of non-elastic tape (Endura Sports Tape, Endura-Tape Pty, Ltd., Australia) was applied.

GS intensity (irradiated muscle activity, viscoelastic components, passive tone) was recorded for three seconds at affected (relaxed) $\mathrm{arm}^{22}$ during maximal voluntary isometric muscle contraction. When the mean maximal voluntary isometric contraction (MVIC) level was reached, irradiated muscle activity was recorded. The sampling rate was $2,000 \mathrm{~Hz}$, with a bandwidth of $20-500 \mathrm{~Hz}$, and the raw data were converted into the root mean square (RMS) data. The RMS was determined based on the EMG signal during that period. Each subject consecutively performed an isometric elbow flexion and extension contraction three times. A thirty second rest period was given between each trial. The activity of two muscles was recorded three times over a period of three seconds. Based on the research methods described by

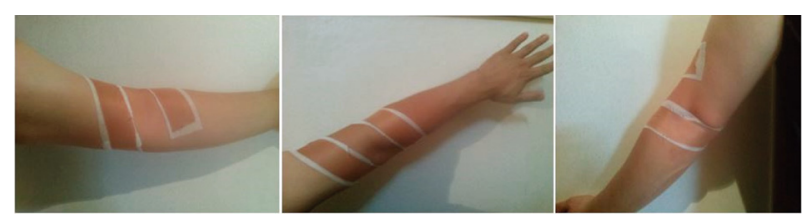

Fig. 1. Non-elastic taping application 
Hwang $^{22)}$, the mean RMS value was determined by averaging the RMS values of the three trials for each task.

The Modified Ashworth Scale (MAS) score was used to evaluate the neurogenic components (active tone) in the flexor muscles of the elbow joint. The MAS uses a graded six-level scale with varying descriptors ranging from 0 (no increase in muscle tone) to 4 (affected parts rigid in flexion or extension $)^{24)}$. The evaluation was performed by a proficient physician who was not blinded to the purpose.

The MAS scores were compared using the Wilcoxon signed-rank test. Categories $1+$ to 4 of the MAS were modified to 2 to 5 for statistical analysis. Differences in the GS intensity between the pre-taping and post-taping conditions were assessed using a paired t-test. Statistical analyses were performed using the SPSS software. The $\mathrm{p}$ values $<0.05$ indicated statistical significance.

\section{RESULTS}

Application of non-elastic tape at the elbow joint significantly changed the GS intensity by contralateral voluntary isometric flexion $(\mathrm{t}(1,13)=3.218, \mathrm{p}=0.007)$, but no significant by extension was identified $(t(1,13)=1.838$, $\mathrm{p}=0.089$ ) (Table 1). Compared to the MAS, the average score of spasticity ranged from 2.29 to 2.14 , which was not found to be statistically significant $(Z=-1.414$, Asymp. Sig. $(2$-tailed $)=0.157)($ Table 2$)$.

\section{DISCUSSION}

Normal upper limb function depends on the ability to statically and dynamically position the limb in an optimal coordinated fashion $^{20}$, but a stroke creates an imbalance between agonist and antagonist muscle pairs. A stoke can also contribute to impairments in passive and/or active elbow motion, resulting in the loss of inhibitory control. This reduction in motion creates functional losses, limits reachable workspace activities, and restricts daily activities ${ }^{11}$.

Weakness in the muscles is commonly seen after a stroke

Table 1. Gs intensity by contralateral maximum voluntary isometric elbow flexion/extension pre and post non elastic taping

\begin{tabular}{lcc}
\hline & \multicolumn{2}{c}{ Gs Intensity } \\
\cline { 2 - 3 } & Pre & Post \\
\hline Elbow flexion* & $1.002 \pm 0.341$ & $0.448 \pm 0.270$ \\
Elbow extension & $1.279 \pm 0.322$ & $0.826 \pm 0.304$ \\
\hline
\end{tabular}

Gs: Global synkinesis

$* \mathrm{p}<0.05$. Values are means $\pm \mathrm{SE}$ and often persists chronically, disrupting the stabilizers of the joint. Weakness of the muscles also often precedes the subsequent development of spasticity ${ }^{27)}$. The hemiplegia has a direct influence on the worsening of neurologic and articular patterns when posturing with a dominant flexor tone in the upper limbs ${ }^{9}$. Therefore, preventing increased abnormal tone and spasticity in a timely manner is essential. To address this concern, the management presented in this paper focuses on elbow flexion hypertonia patterns. This pattern of excessive elbow flexion can worsen with walking (e.g., increased flexor posturing with walking or running), emotional excitement, or with the use of the uninvolved extremity for activities such as getting dressed, washing one's hands, or writing ${ }^{11)}$. The management of abnormal flexor muscle tone of the elbow in stroke patients is considered an important factor for functional recovery ${ }^{2}$.

In a study described by Castilho ${ }^{25}$, researchers applied neural mobilization of the upper limb contralateral to the hemiplegia and found a reduction in electromyographic activity in the biceps brachii but found an increase in electromyographic activity when the process of capturing the signals was performed with the arm in extension. They also report that the central and peripheral nervous systems are considered a single continuous system and that any limb movement has mechanical consequences in the neuro axis. This finding suggests that contralateral limb movements influence the affected limb.

The principal aim of this study was to evaluate the effect of non-elastic tape on an elbow flexor muscle in hemiplegic patients that was measured by GS intensity and MAS. In our study, non-elastic tape was applied in a spiral manner across the muscle belly and along the elbow extension and forearm when in the supinated position. This was done to inhibit the flexor tone that is associated with GS. As a result, the GS intensity was significantly decreased in contralateral isometric flexion. According to a study completed by Hwang ${ }^{22)}$, GS activity was observed to spread through the muscles of the contralateral upper extremity in association with target movements. The level of GS intensity in the paretic arm related to the functional outcome of post-stroke patients ${ }^{23)}$ and was directly linked to spastic resistance and angular velocity mirrored in their elbow flexor tone ${ }^{28)}$; this was especially true when GS was triggered by contraction of the contralateral elbow flexors ${ }^{22}$. Studies using electromyography (EMG) in hemiparetic subjects show that EMG levels were significantly higher during contralateral upper extremity tasks. The neurophysiological mechanisms involved demonstrated a decrease of cortical inhibition acting on the spinal cord ${ }^{22}$.

Taping techniques can be used as an adjunct during the rehabilitation process to enhance functional recovery by improving alignment, stimulating or inhibiting muscle

Table 2. Clinical assessment of spastic elbow flexor pre and post non-elastic taping using the MAS

\begin{tabular}{|c|c|c|c|c|c|c|c|c|c|c|c|c|c|c|}
\hline \multirow{2}{*}{$\begin{array}{l}\text { MAS } \\
\text { core }\end{array}$} & \multicolumn{14}{|c|}{ Subject } \\
\hline & $A$ & B & $\mathrm{C}$ & $\mathrm{D}$ & $\mathrm{E}$ & $\mathrm{F}$ & $\mathrm{G}$ & $\mathrm{H}$ & I & $\mathrm{J}$ & $\mathrm{K}$ & $\mathrm{L}$ & $M$ & $\mathrm{~N}$ \\
\hline Pre & 2 & 2 & 1 & 3 & 3 & 1 & 2 & 3 & 3 & 3 & 3 & 3 & 3 & 2 \\
\hline Post & 2 & 2 & 1 & 3 & 2 & 1 & 2 & 2 & 3 & 3 & 2 & 3 & 2 & 2 \\
\hline
\end{tabular}


function, and improving proprioceptive function of the joint structure ${ }^{12)}$. Proper taping is a useful adjunct to these processes and has the particular advantage of lasting well beyond patient-therapist contact, thus extending the duration of therapy ${ }^{20)}$. Following the applied direction, taping perpendicular to a muscle inhibits activity and taping parallel to a muscle promotes activity ${ }^{14,27)}$. The inhibitory effects of this study could be explained on the basis of cutaneous effects being produced by laying the tape upon the skin. Cutaneous afferents have the ability to both facilitate and inhibit local motoneurone pool excitability ${ }^{14)}$. And they suggest that if the muscle is held by the tension of the tape, a reduction in tonic muscle spindle activity may result. This would reduce the spindle afferent input upon the motoneurone pool, which may lead to its inhibition. The mechanisms of cutaneous mechanoreceptors have been explained in several studies ${ }^{8,17-19,29,30)}$, and most studies consider taping the skin to be an effective way to stimulate cutaneous mechanoreceptors, thereby allowing more sensory signals to be carried to the central nervous system for information integration $^{31,32)}$. Recent studies have documented increasing proprioceptive function resulting from the cutaneous afferent stimulation of the $\operatorname{skin}^{17,31)}$. Skin sensation plays an important role in detecting joint position and movement ${ }^{31)}$; proprioception refers to mechanoreceptor detect information regarding joint position, movement, and the perception of these movements by the central nervous system ${ }^{32}$. Afferent input from the joint capsule, ligaments, and muscle spindles to allow the central nervous system (CNS) to build precise proprioceptive information and to induce changes in local muscle tone. Other proposed mechanisms include the biomechanical realignment of a joint. $\mathrm{Chou}^{21)}$ found that the application of the non-elastic tape provided proper body alignment and suggestss that this method contributes to improve stability, improved proprioception, and neuromuscular control ${ }^{33)}$. The affected limb is positioned opposite to the direction of muscle spasticity. Taping is used to maintain the joint in an appropriate anatomic position to prevent or reduce positioning default ${ }^{27)}$. It also helps to decrease the stress on the joint and prevent subluxation by reducing the gravitational pull on the joint. ElKhatib ${ }^{26)}$ suggests that the position of the upper extremity following a stroke affects not only the patients' ability to reach, hold, and manipulate an object but also their ability to stand up and walk; therefore, taping can lead to improvements in the upper extremity after the stroke. Kneeshaw ${ }^{34)}$ suggests that taping from the onset of stroke until restoration of muscle tone may prevent the onset of hemiplegic pain by enforcing proper positioning. Recent studies have shown that the pull involved in applying the second of the two tapes is critical to the electromyographic and mechanical positional changes observed during successful taping application ${ }^{20}$. Likewise, Someeh ${ }^{33)}$ showed that repositioning non-elastic tape can significantly improve postural control in healthy subjects and can be applied immediately prior to increase joint awareness. In addition, the repetitive feedback formation of the cerebrum through the taping triggers a decrease in movement related cortical potential (MRCP), positively influencing functional movements ${ }^{35)}$.

In patients with stroke, neuronal irradiation was manifested and mutually coupled with the flexor muscles of the affected upper limb ${ }^{22}$. Although the specific pathophysiological mechanisms underlying the development of spasticity are not fully understood, evidence suggests that abnormalities in spinal pathways regulating the stretch reflex may contribute to the hypertonia and hyperreflexia that characterize spasticity ${ }^{9}$. The technique of positioning a limb in a reflex-inhibiting pattern can help to prevent inefficient movement and maintain muscle tone ${ }^{27}$ ). We supposed that proper joint position through non-elastic tape provides adequate joint stability and proprioceptive information feedback and can also contribute to changes in local muscle tone.

The MAS scale showed no significant MAS grades associated with a significant decrease in passive range of motion (PROM) at the wrist and elbow, particularly in those who had decreased extension due to the greater relative strength in the flexor muscles. Although the Ashworth Scale measures hypertonia by gauging the resistance to passive displacement of the limb, Pizzi ${ }^{6}$ suggested that the MAS does not provide a valid measure of spasticity at lower grades, especially between the 1 and $1+$ grades of the scale; the MAS score is quite low in the present study. Furthermore, we have concluded that changes in the active tone are not significant enough.

We must consider the function of the shoulder, the elbow, and the wrist but our study focused only on the elbow joint. Although the non-elastic tape contributed to a decrease in abnormal passive flexor tone, we have a small sample size, and the mechanism is are not clear. Thus, there is a need for future studies dealing to a greater degree with abnormal muscle tone.

These results may be used to guide the choice of appropriate interventions, such as those involving muscle tone and practices to prevent spastic elbow flexion.

\section{REFERENCES}

1) Niessen $\mathrm{MH}$, Veeger $\mathrm{DH}$, Meskers CG, et al.: Relationship among shoulder proprioception, kinematics, and pain after stroke. Arch Phys Med Rehabil, 2009, 90: 1557-1564. [Medline] [CrossRef]

2) Ohn SH, Yoo WK, Kim DY, et al.: Measurement of synergy and spasticity during functional movement of the post-stoke hemiplegic upper limb. J Electromyogr Kinesiol, 2013, 23: 501-507. [Medline] [CrossRef]

3) Jung YJ, Hong JH, Kwon HG, et al.: The effect of a stretching device on hand spasticity in chronic hemiparetic stroke patients. NeuroRehabilitation, 2011, 29: 53-59. [Medline]

4) Donec V, Varžaitytė L, Kriščiūnas A: The effect of Kinesio Taping on maximal grip force and key pinch force. Pol Ann Med, 2012, 19: 98-105. [CrossRef]

5) Boudarham J, Roche N, Teixeira M, et al.: Relationship between neuromuscular fatigue and spasticity in chronic stroke patients: a pilot study. J Electromyogr Kinesiol, 2014, 24: 292-299. [Medline] [CrossRef]

6) Pizzi A, Carlucci G, Falsini C, et al.: Evaluation of upper-limb spasticity after stroke: a clinical and neurophysiologic study. Arch Phys Med Rehabil, 2005, 86: 410-415. [Medline] [CrossRef]

7) Bunata R, Icenogle K: Cerebral palsy of the elbow and forearm. J Hand Surg Am, 2014, 39: 1425-1432. [Medline] [CrossRef]

8) Gómez-Soriano J, Abián-Vicén J, Aparicio-García C, et al.: The effects of Kinesio taping on muscle tone in healthy subjects: a double-blind, placebocontrolled crossover trial. Man Ther, 2014, 19: 131-136. [Medline] [CrossRef]

9) Motta-Oishi AA, Magalhães FH, Mícolis de Azevedo F: Neuromuscular electrical stimulation for stroke rehabilitation: is spinal plasticity a possible mechanism associated with diminished spasticity? Med Hypotheses, 2013, 81: 784-788. [Medline] [CrossRef]

10) Bae SH, Kim KY: Effects of vibration stimulation method on upper limbs 
spasticity in patients with brain lesion. Journal of the Korea AcademiaIndustrial Cooperation Society, 2011, 12: 3109-3116. [CrossRef]

11) Barus D, Kozin SH: The evaluation and treatment of elbow dysfunction secondary to spasticity and paralysis. J Hand Ther, 2006, 19: 192-205. [Medline] [CrossRef]

12) Jaraczewska E, Long C: Kinesio taping in stroke: improving functional use of the upper extremity in hemiplegia. Top Stroke Rehabil, 2006, 13: 31-42. [Medline] [CrossRef]

13) Martin $\mathrm{T}$ : The use of Kinesio Tex ${ }^{\circledR}$ tape as an adjunct to treatment for children with brachial plexus injury. UPBN Outreach, 2005, 17: 8-10.

14) Alexander CM, Stynes $S$, Thomas $A$, et al.: Does tape facilitate or inhibit the lower fibres of trapezius? Man Ther, 2003, 8: 37-41. [Medline] [CrossRef]

15) Lee DH, Kim WJ, Oh JS, et al.: Taping of the elbow extensor muscle in chronic stroke patients: comparison between before and after three-dimensional motion analysis. J Phys Ther Sci, 2015, 27: 2101-2103. [Medline] [CrossRef]

16) Kim EB, Kim YD: Effects of kinesiology taping on the upper-extremity function and activities of daily living in patients with hemiplegia. J Phys Ther Sci, 2015, 27: 1455-1457. [Medline] [CrossRef]

17) Alexander CM, McMullan M, Harrison PJ: What is the effect of taping along or across a muscle on motoneurone excitability? A study using triceps surae. Man Ther, 2008, 13: 57-62. [Medline] [CrossRef]

18) Campolo M, Babu J, Dmochowska K, et al.: A comparison of two taping techniques (kinesio and mcconnell) and their effect on anterior knee pain during functional activities. Int J Sports Phys Ther, 2013, 8: 105-110. [Medline]

19) Kang MH, Choi SH, Oh JS: Postural taping applied to the low back influences kinematics and EMG activity during patient transfer in physica therapists with chronic low back pain. J Electromyogr Kinesiol, 2013, 23: 787-793. [Medline] [CrossRef]

20) Morrissey D: Proprioceptive shoulder taping. J Bodyw Mov Ther, 2000, 4 189-194. [CrossRef]

21) Chou E, Kim KM, Baker AG, et al.: Lower leg neuromuscular changes following fibular reposition taping in individuals with chronic ankle instability. Man Ther, 2013, 18: 316-320. [Medline] [CrossRef]

22) Hwang IS, Tung LC, Yang JF, et al.: Electromyographic analyses of globa synkinesis in the paretic upper limb after stroke. Phys Ther, 2005, 85: 755-765. [Medline]

23) Boissy P, Bourbonnais D, Gravel D, et al.: Effects of upper and lower limb static exertions on global synkineses in hemiparetic subjects. Clin Rehabil, 2000, 14: 393-401. [Medline] [CrossRef]

24) Doucet BM, Mettler JA: Effects of a dynamic progressive orthotic intervention for chronic hemiplegia: a case series. J Hand Ther, 2013, 26: 139-146, quiz 147. [Medline] [CrossRef]

25) Castilho J, Ferreira LA, Pereira WM, et al.: Analysis of electromyographic activity in spastic biceps brachii muscle following neural mobilization. J Bodyw Mov Ther, 2012, 16: 364-368. [Medline] [CrossRef]

26) ElKhatib RS, ElNegmy EH, Salem AH, et al.: Kinesio arm taping as prophylaxis against the development of Erb's Engram. J Adv Res, 2013, 4: 485-491. [Medline] [CrossRef]

27) Vasudevan JM, Browne BJ: Hemiplegic shoulder pain: an approach to diagnosis and management. Phys Med Rehabil Clin N Am, 2014, 25: 411437. [Medline] [CrossRef]

28) Dvir Z, Panturin E: Measurement of spasticity and associated reactions in stroke patients before and after physiotherapeutic intervention. Clin Rehabil, 1993, 7: 15-21. [CrossRef]

29) Park J, Kim K: Initial effects of the non-elastic taping technique on grip strength and EMG in female with lateral epicondylalgia. J The Korean Soc Phys Med, 2012, 7: 525-533. [CrossRef]

30) Kim WI, Choi YK, Lee JH, et al.: The effect of muscle facilitation using kinesio taping on walking and balance of stroke patients. J Phys Ther Sci, 2014, 26: 1831-1834. [Medline] [CrossRef]

31) Chang HY, Chou KY, Lin JJ, et al.: Immediate effect of forearm Kinesio taping on maximal grip strength and force sense in healthy collegiate athletes. Phys Ther Sport, 2010, 11: 122-127. [Medline] [CrossRef]

32) Gusella A, Bettuolo M, Contiero F, et al.: Kinesiologic taping and muscular activity: a myofascial hypothesis and a randomised, blinded trial on healthy individuals. J Bodyw Mov Ther, 2014, 18: 405-411. [Medline] [CrossRef]

33) Someeh M, Norasteh AA, Daneshmandi H, et al.: Immediate effects of Mulligan's fibular repositioning taping on postural control in athletes with and without chronic ankle instability. Phys Ther Sport, 2015, 16: 135-139. [Medline] [CrossRef]

34) Kneeshaw D: Shoulder taping in the clinical setting. J Bodyw Mov Ther 2002, 6: 2-8. [CrossRef]

35) Bae SH, Lee JH, Oh KA, et al.: The effects of kinesio taping on potential in chronic low back pain patients anticipatory postural control and cerebral cortex. J Phys Ther Sci, 2013, 25: 1367-1371. [Medline] [CrossRef] 